\title{
Anaphylaxis to Ciprofloxacin Requiring Emergent Surgical Cricothyrotomy
}

\author{
Wesley Tang, Edwina Rao \\ Department of Medicine, Kettering Medical Center, Kettering, OH, USA
}

Received: 11/01/2022

Accepted: 27/01/2022

Published: 24/02/2022

How to cite this article: Tang W, Rao E. Anaphylaxis to ciprofloxacin requiring emergent surgical cricothyrotomy. EJCRIM 2022;9:doi:10.12890/2022_003180.

Conflicts of Interests: The Authors declare that there are no competing interests.

This article is licensed under a Commons Attribution Non-Commercial 4.0 License

\section{ABSTRACT}

Although allergies to antibiotics are commonly stated, anaphylactic reactions are uncommonly reported. This is especially the case with reactions to fluoroquinolone antibiotics. Furthermore, airway emergencies are rare. We present a case of ciprofloxacin-induced acute airway obstruction and anaphylaxis, necessitating emergent surgical cricothyrotomy following respiratory distress.

\section{KEYWORDS}

Fluoroquinolones, ciprofloxacin, emergent airway, cricothyrotomy, anaphylaxis

\section{LEARNING POINTS}

- Anaphylactic reactions to fluoroquinolone antibiotics are extremely rare.

- There are currently no standardized diagnostic tests, making the diagnosis of anaphylaxis challenging and largely based on history alone.

- Treating clinicians should be aware of this rare, but potentially rapidly fatal adverse drug reaction.

\section{INTRODUCTION}

Allergies to antibiotics are common, with roughly $2.2 \%$ of all hospitalized patients developing a cutaneous drug reaction ${ }^{[1]}$. The majority of these reactions are caused by trimethoprim-sulfamethoxazole, amoxicillin, penicillin and ampicillin ${ }^{[2]}$. Fluoroquinolones are a class of antibiotics with most reactions being mainly mild and affecting the gastrointestinal tract or central nervous system ${ }^{[3]}$. The estimated prevalence of hypersensitivity reactions to fluoroquinolones of any severity has been estimated to be low at 44 emergency department visits per 100,000 prescriptions in one study in the USA ${ }^{[4]}$. This same study identified higher rates of moxifloxacin-related hypersensitivity reactions compared with levofloxacin or ciprofloxacin ${ }^{[4]}$. The authors present an unusual case of a patient who took one dose of oral ciprofloxacin for epididymitis on an outpatient basis. Within 1 hour of ingestion, the patient complained of difficulty speaking, and severe swelling of the eyes, lips and tongue. After failed attempts at endotracheal intubation, an emergent surgical cricothyroidotomy was performed.

\section{CASE DESCRIPTION}

A 50-year-old man with a past medical history of morbid obesity (Body Mass Index of $57.6 \mathrm{~kg} / \mathrm{m}^{2}$ ), obstructive sleep apnoea, paroxysmal atrial fibrillation, hypertension and diet-controlled type II diabetes mellitus, presented to the emergency department with a chief complaint of difficulty breathing and chest tightness. Home medications included apixaban, carvedilol and metformin. Notably, the patient had never been on an angiotensin-converting enzyme inhibitor (ACEI) or angiotensin receptor blocker (ARB). The patient had been prescribed a course of ciprofloxacin by his outpatient primary care provider (PCP) for the first time earlier that evening for epididymitis. His family members noted that the patient began having severe facial and tongue swelling, and called emergency medical services. Paramedics arrived at the 
scene and called with prehospital notification of respiratory distress and administered $50 \mathrm{mg}$ diphenhydramine and $0.5 \mathrm{mg}$ intramuscular epinephrine on route to the hospital. By the time the patient arrived at the hospital, he could not speak and was pointing to his chest. His temperature was $36.94^{\circ} \mathrm{C}$, blood pressure was $204 / 111 \mathrm{mmHg}$, pulse was $78 \mathrm{bpm}$, respiratory rate was up to 38 breaths per minute, and oxygen saturation was $94 \%$. On physical examination, the patient was alert, appeared anxious and in distress, was unable to speak, and was noted to have an obese body habitus. He had bilateral periorbital oedema, and his lips and tongue were swollen. The posterior oropharynx could not be visualized. He was using accessory muscles to breathe, and stridor was noted. He was wheezing throughout all lung fields and poor air movement was noted. Heart sounds had a regular rate and rhythm. The patient had an obese abdomen that was non-tender to palpation. The skin was diaphoretic. Distal pulses were intact, and no cyanosis or lower extremity oedema was noted. He had full strength in all extremities.

The patient was treated with inhaled ipratropium bromide/albuterol, nebulized racemic epinephrine, 10 mg intravenous dexamethasone, 20 $\mathrm{mg}$ intravenous famotidine and $0.3 \mathrm{mg}$ intramuscular epinephrine, and was started on an epinephrine drip. However, as his symptoms were not improving, he began to develop respiratory fatigue, severe stridor, and an inability to control his secretions. Endotracheal intubation was attempted with video laryngoscopy; however, the camera could not be passed over the patient's swollen tongue and the endotracheal tube could not be placed due to upper airway and pharynx oedema. The patient then underwent emergent bedside cricothyrotomy, which was successful.

Potassium was $3.0 \mathrm{mmol} / \mathrm{l}$ and was repleted. Troponins were not elevated. The serum and urine toxicity screens were negative. White blood cells and haemoglobin were within normal limits. The electrocardiogram was non-acute. A computed tomography (CT) scan of the neck showed extensive subcutaneous stranding and swelling throughout the periorbital region, face and neck. Extensive soft tissue swelling and secretions throughout the visualized airway were also noted (Fig. 1). CT of the head showed extensive pre-septal periorbital soft tissue oedema bilaterally (Fig. 2).

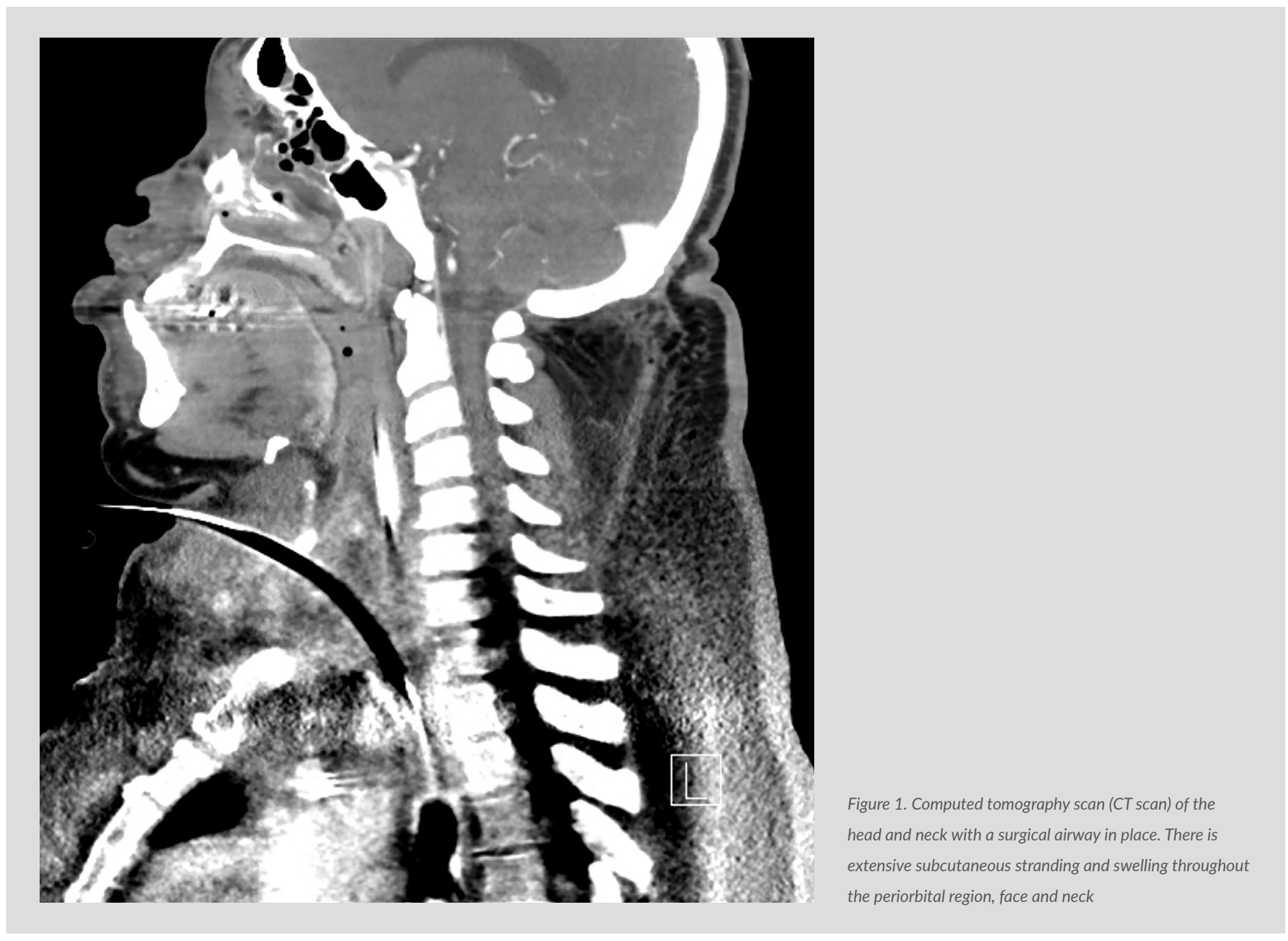




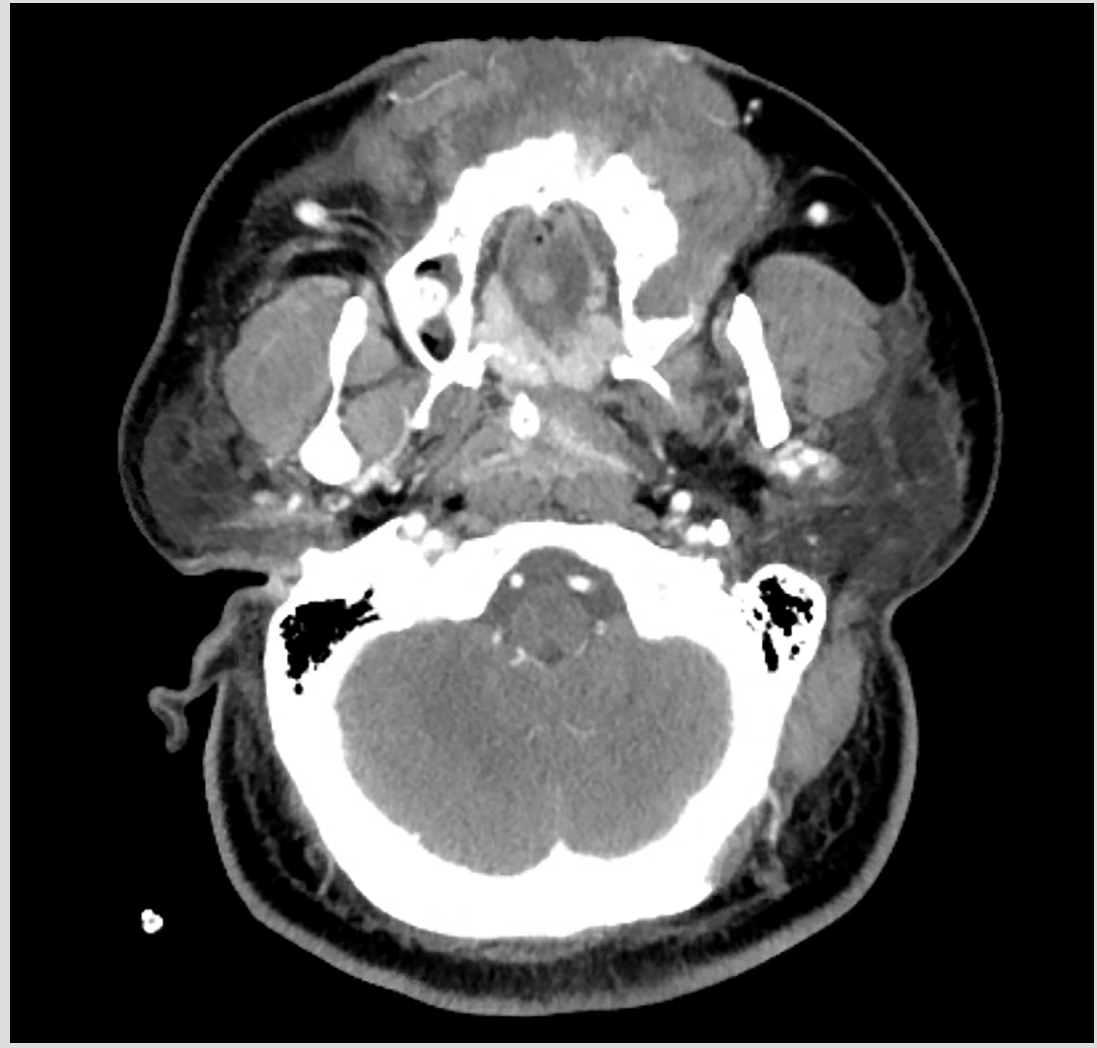

Figure 2. Computed tomography scan (CT scan) of the head. There is extensive subcutaneous stranding and swelling throughout the periorbital region, face and neck

The patient was transferred to the intensive care unit for further care. Respiratory therapy and continued mechanical ventilation were provided. He was continued on epinephrine infusion and was placed on IV steroids and antihistamines. He was not given any further doses of ciprofloxacin and was switched to doxycycline for epididymitis. He was taken to the operating room on hospital day 3 for attempted extubation and underwent pharyngoscopy. However, after evaluation of his airway by Anesthesia and Otorhinolaryngology, it was felt it was unsafe to proceed given the minimal reduction in airway swelling. The patient was subsequently taken back to the operating room on hospital day 5 for high-risk extubation and formal tracheostomy. He tolerated the procedure well and there were no complications. Steroids were weaned on hospital day 7. The patient improved and underwent decannulation on hospital day 16. On the day of discharge, the patient was alert and oriented to person, place, time and situation, was able to follow three-step commands, and was able to walk 150 feet independently. His tongue swelling, lip swelling and periorbital oedema were resolved. His epididymitis had resolved. His lungs were clear to auscultation bilaterally, and his breathing was not laboured. The tracheostomy site was clear and dry with the dressing in place. His home medications of metformin, carvedilol and apixaban were restarted days before discharge and without recurrence of symptoms. In further history taking, the patient indicated that his medications were stable for the last year prior to presentation and that he had not eaten anything out of the ordinary. He denied recent exposure to chemicals, changes to his normal routine, or changes to his home or work environment. The patient followed up with both Otorhinolaryngology and Pulmonology within 4 weeks of discharge and had experienced no acute issues. He continues to follow up with his PCP and has had no recurrence 6 months after hospital discharge.

\section{DISCUSSION}

To date, the exact mechanism of fluoroquinolone allergy has not been clearly defined. Furthermore, there are no standardized diagnostic tests, making the diagnosis challenging and largely based on history alone ${ }^{[5]}$. Skin testing has been shown to have false-positive results with fluoroquinolones when used in high concentrations ${ }^{[6]}$. Other studies have shown fluoroquinolones to directly induce histamine release ${ }^{[7]}$. One other option is the basophil activation test, although this has shown varying testing characteristics with fluoroquinolones with reported sensitivity ranging from $50 \%$ to $100 \%$, and specificity from $80 \%$ to $100 \%{ }^{[8,9]}$. Finally, if other diagnostic tests or procedures lead to inconclusive results, there is also drug provocation testing. However, drug provocation tests have serious drawbacks as they are often not performed, are time-consuming, require trained personnel in a clinical setting, and may provoke life-threatening reactions ${ }^{[10]}$. 
Drug hypersensitivity reactions to fluoroquinolones are becoming more common due to increasing prevalence and case complexity. One study from 2005-2010 estimated an increase in reported allergy to fluoroquinolones from 0.5\% to 6.8\% diagnosed by clinical history, skin tests, in vitro tests, and drug provocation testing ${ }^{[11]}$. In 2016, the United States Food and Drug Administration (US FDA) updated the boxed warning on fluoroquinolones recommending against their use as first-line agents for the routine pharmacological management of uncomplicated urinary tract infections, acute sinusitis and acute bronchitis. Interestingly, and despite the US FDA's boxed warning, there was no significant change in fluoroquinolone prescription rates before and after the FDA boxed warning ${ }^{[12]}$.

In summary, we report a case of severe anaphylaxis induced by ciprofloxacin necessitating an emergent surgical airway. Clinicians should be aware of this as a rare, but potentially fatal adverse drug reaction.

\section{REFERENCES}

1. Gruchalla RS, Pirmohamed M. Clinical practice. Antibiotic allergy. N Engl J Med 2006;354(6):601-609.

2. Bigby M, Jick S, Jick H, Arndt K. Drug-induced cutaneous reactions. A report from the Boston Collaborative Drug Surveillance Program on 15,438 consecutive inpatients, 1975 to 1982. JAMA 1986;256(24):3358-3363.

3. Bertino J, Jr., Fish D. The safety profile of the fluoroquinolones. Clin Ther 2000:22(7):798-817; discussion 797.

4. Jones SC, Budnitz DS, Sorbello A, Mehta H. US-based emergency department visits for fluoroquinolone-associated hypersensitivity reactions. Pharmacoepidemiol Drug Saf 2013;22(10):1099-1106.

5. Demir S, Gelincik A, Akdeniz N, Aktas-Cetin E, Olgac M, Unal D, et al. Usefulness of in vivo and in vitro diagnostic tests in the diagnosis of hypersensitivity reactions to quinolones and in the evaluation of cross-reactivity: a comprehensive study including the latest quinolone gemifloxacin. Allergy Asthma Immunol Res 2017;9(4):347-359.

6. Brockow K, Garvey LH, Aberer W, Atanaskovic-Markovic M, Barbaud A, Bilo MB, et al. Skin test concentrations for systemically administered drugs - an ENDA/EAACI Drug Allergy Interest Group position paper. Allergy 2013;68(6):702-712.

7. Seitz CS, Bröcker EB, Trautmann A. Diagnostic testing in suspected fluoroquinolone hypersensitivity. Clin Exp Allergy 2009;39(11):1738-1745.

8. Lobera T, Audícana MT, Alarcón E, Longo N, Navarro B, Muñoz D. Allergy to quinolones: low cross-reactivity to levofloxacin. J Investig Allergol Clin Immunol 2010;20(7):607611.

9. Ben Said B, Berard F, Bienvenu J, Nicolas JF, Rozieres A. Usefulness of basophil activation tests for the diagnosis of IgE-mediated allergy to quinolones. Allergy 2010;65(4):535536.

10. Doña I, Moreno E, Pérez-Sánchez N, Andreu I, Hernández Fernandez de Rojas D, Torres MJ. Update on quinolone allergy. Curr Allergy Asthma Rep 2017;17(8):56.

11. Doña I, Blanca-López N, Torres MJ, García-Campos J, García-Núñez I, Gómez F, et al. Drug hypersensitivity reactions: response patterns, drug involved, and temporal variations in a large series of patients. J Investig Allergol Clin Immunol 2012;22(5):363-371.

12. Bratsman A, Mathias K, Laubscher R, Grigoryan L, Rose S. Outpatient fluoroquinolone prescribing patterns before and after US FDA boxed warning. Pharmacoepidemiol Drug Saf 2020;29(6):701-707. 